\title{
Accuracy assessment of lunar topography models
}

\author{
H. S. Fok ${ }^{1}$, C. K. Shum ${ }^{1}$, Yuchan $\mathrm{Yi}^{1}$, Hiroshi Araki ${ }^{2}$, Jinsong Ping ${ }^{3}$, James G. Williams ${ }^{4}$, Georgia Fotopoulos ${ }^{5}$, \\ Hirotomo Noda ${ }^{2}$, Sander Goossens $^{2}$, Qian Huang ${ }^{3}$, Yoshiaki Ishihara ${ }^{2}$, Koji Matsumoto $^{2}$, \\ Jürgen Oberst $^{6}$, and Sho Sasaki ${ }^{2}$ \\ ${ }^{1}$ Geodetic Science, School of Earth Sciences, The Ohio State University, 125 South Oval Mall, \\ 275 Mendenhall Lab., Columbus, OH 43210, USA \\ ${ }^{2}$ RISE Project, National Astronomical Observatory of Japan, 2-12, Hoshigaoka, Mizusawa, Oshu, Iwate 023-0861, Japan \\ ${ }^{3}$ Shanghai Astronomical Observatory, Chinese Academy of Sciences, Nandan Road 80, Shanghai, China, 200030 \\ ${ }^{4}$ Jet Propulsion Laboratory, California Institute of Technology, MS 238-600, 4800 Oak Grove Drive, Pasadena, CA, 91109, USA \\ ${ }^{5}$ Dept. of Geosciences, The University of Texas at Dallas, 800 West Campbell Rd, Richardson, TX 75080-3021, USA \\ ${ }^{6}$ German Aerospace Center (DLR), Institute of Planetary Research, Rutherfordstraße 2, 12489 Berlin, Germany
}

(Received June 29, 2009; Revised June 22, 2010; Accepted August 12, 2010; Online published February 21, 2011)

Both the Chang'E-1 and SELenological and ENgineering Explorer (SELENE) lunar missions, launched in 2007, provide an opportunity for signi cant advances in lunar science. In particular, both orbiters provide re ned lunar topography models with unprecedented ner resolution and improved accuracy, especially for the far side and the polar areas of the Moon where fewer valid measurements have been available to date. An evaluation of the derived topography models is essential for the improved interpretation of selenophysics, including a knowledge of the interior of the Moon. This study provides both external and internal accuracy assessments for the derived topographic models (note that different data sets are used, albeit independent). The external comparison, which consists of comparing the topographic models with landmarks established with lunar laser ranging and radio tracking, yields an accuracy estimate of $157 \mathrm{~m}$ for the Change'E-1 model and $58 \mathrm{~m}$ for the SELENE model (150 $\mathrm{m}$ and $55 \mathrm{~m}$ if the newly recovered Lunakhod-1 site is included). The internal comparison, consisting of crossover analysis of the altimeter data after the removal of once-per-orbital-revolution errors, yields an accuracy estimate of $206 \mathrm{~m}$ and $68 \mathrm{~m}$, respectively. These comparisons allow the establishment of conservative estimates of accuracy of $200 \mathrm{~m}$ and $70 \mathrm{~m}$ for the Chang'E-1 and SELENE models, respectively. Given the conservative estimates on the accuracy, both models yield signi cant improvement by factors of 2.5 and 8 , respectively, when compared with a contemporary lunar topography model, i.e., the Uni ed Lunar Control Network 2005 (ULCN2005).

Key words: Accuracy assessment, lunar topography, selenodesy, Chang'E-1, SELENE.

\section{Introduction}

Since the 1960s, numerous lunar missions, such as the Apollo missions from the USA and missions from the former Soviet Union, have been launched to explore the Moon. Various geodetic measurements, such as experimental laser altimeter ranging (Sjogren and Wollenhaupt, 1973) and the Deep Space Network radio tracking of spacecraft, were among the early lunar observations. Additional contributions include the robotic and human placement of laser retrore ector arrays at selected locations on the surface of the Moon. The retrore ectors enable highly accurate range measurements of the Earth-Moon distance by Lunar Laser Ranging (LLR) and have stimulated scienti c research in a broad variety of elds, including astronomy, relativistic and gravitational physics, selenodesy, and selenodynamics (Dickey et al., 1994).

Size and shape are the fundamental parameters used to describe any extraterrestrial planetary body. To realize these quantities, it is essential to measure the topography

Copyright (C) The Society of Geomagnetism and Earth, Planetary and Space Sciences (SGEPSS); The Seismological Society of Japan; The Volcanological Society of Japan; The Geodetic Society of Japan; The Japanese Society for Planetary Sciences; TERRAPUB.

doi: $10.5047 /$ eps.2010.08.005 of the planetary body in the center-of-mass (CoM) reference frame (Smith et al., 1997). The U.S. Department of Defense Clementine mission on January 25, 1994 was the rst U.S. spacecraft to be launched to the Moon in over 20 years (since Explorer 49 in June 1973). One signi cant accomplishment of the Clementine mission was the laser altimeter mapping of the lunar topography, which resulted in an improved knowledge of the Moon's internal structure and its thermal history (Zuber et al., 1994). The topography measurement of a planetary body, when combined with gravity (and appropriate assumptions), enables quanti cation of not only the size and shape of the planetary body, but also of critical geophysical parameters, such as crustal thickness and subsurface density anomalies. Accurate topography data also provide information on illumination conditions that can be used to identify polar areas in permanent sunlight and cold traps, which may contain water ice (Wieczorek, 2007; Noda et al., 2008; Ping et al., 2009).

Only a few valid measurements were made on the lunar far side in previous studies due to terrain roughness, which results in relatively complex return pulses that are more dif cult to interpret (if at all) (Zuber et al., 1994; Wieczorek et al., 2006). Also, because Doppler track- 
ing signals are interrupted while a spacecraft orbits behind the Moon, the orbits over the far side of the Moon were less well-determined (Namiki et al., 1999). As a consequence, radial uncertainties of several hundred meters exist in the derived lunar topography of the Uni ed Lunar Control Network 2005 (ULCN2005) control network (Archinal et al., 2007; Araki et al., 2009). Also, no topographic data were available for the polar areas, which were beyond reach for the Clementine spacecraft in its eccentric $425 \mathrm{~km} \times 2950 \mathrm{~km}$ orbit.

In 2007, more than a decade after the 1994 Clementine mission, two lunar satellite missions, Chang'E-1 and SELenological and ENgineering Explorer (SELENE) were launched by China and Japan, respectively, with the goal to provide measurements with comprehensive spatial coverage and to further advance lunar sciences. The Indian lunar mission, called 'Chandrayaan-1', followed closely in 2008. In particular, both Chang'E-1 and SELENE carry laser altimeters and are able to derive topography models using their respective altimeter range measurements.

This study provides an accuracy assessment of the SELENE Laser Altimeter (LALT)- and the Chang'E-1 Laser Altimeter Mission (LAM)-determined lunar topography models. Both topography models and the ULCN2005 model are compared with coordinates of the in-situ LLR Retrore ector (LLRR) sites (Apollo 11, 14, 15, and the Soviet sites Lunakhod 1, newly recovered in April 2010, and Lunakhod 2) and the radio-tracked Apollo Lunar Surface Experiments Package (ALSEP) sites (Apollo 12, 14, 15,16 , and 17) using statistical analysis. The LLRR reference sites, which are located in the equatorial region on the lunar near side, have been observed for over three decades with suf cient accuracy through LLR. Because the low-altitude lunar polar orbiters undergo large periodic perturbations in eccentricity and inclination (up to $\pm 1^{\circ}$ ) that generate crossovers, the locations of crossovers for both SELENE and Chang'E-1 surface tracks are concentrated near the polar region. In this study, single-satellite altimeter crossover analysis (Shum et al., 1990) (SELENE crossovers and Chang'E-1 crossovers) is conducted to evaluate the orbit accuracy for the respective satellite orbits, which directly assesses the accuracy of the LALT- and LAM-determined lunar topography models. As a result, these two assessment schemes complement each other in terms of spatial location. In the following sections, we demonstrate that the datasets exceed the accuracy of the contemporary lunar model and that they may potentially be improved by the crossover analysis.

\section{Data Used in the Study}

\subsection{Lunar laser altimeter data and derived topogra- phy}

SELENE, launched by the H-IIA rocket at Tanegashima Space Center in Japan on September 14, 2007, consists of a main lunar orbiting satellite in a polar orbit with an inclination of $90^{\circ} \pm 1^{\circ}$ at an approximate altitude of $100 \mathrm{~km}$ and of two sub-satellites (called RSTAR and VSTAR) in elliptical polar orbits at initial apocenter altitudes of 2,400 km and $800 \mathrm{~km}$, respectively, and at initial pericenter altitudes of $100 \mathrm{~km}$ above the lunar surface. RSTAR is a transponding satellite for the four-way Doppler tracking with the main satellite, whereas VSTAR is a sub-satellite used for Very Long Baseline Interferometry (VLBI) tracking together with RSTAR. The functions of those two subsatellites enrich our knowledge of both the terrain and gravity eld on the far side of the Moon, which was previously not observed (Araki et al., 2008; Kikuchi et al., 2009; Namiki et al., 2009). Data collection began on December 30, 2007.

Chang'E-1, launched by the Long March 3A rocket from Xichang Satellite Launch Center in China on October 24, 2007 , comprises one satellite in a 2 -h polar orbit with an inclination of $90^{\circ} \pm 2^{\circ}$ at an altitude of $200 \mathrm{~km}$. It began collecting data on November 27, 2007 (Ping et al., 2009). The precise orbit of Chang'E-1 is primarily determined using the close-loop two-way Uni ed S-band (USB) Doppler and Ranging data and the VLBI delay and delay rate data collected by four Earth-based VLBI stations. A comparison of the two missions and their similar orbit con gurations allows an independent validation of the lunar topography models.

The laser altimeters on the SELENE main satellite (LALT) and Chang'E-1 satellite (LAM) generate topographic measurements with an improved accuracy and spatial resolution compared with previous ones. The precision of the LALT and LAM laser altimeters with $3 \sigma$ are claimed to be $\pm 4 \mathrm{~m}$ and $\pm 5 \mathrm{~m}$, respectively. The altimeter data records of both missions contain time tags at each three-dimensional (3-D) location on the Moon, with attitude and range measurements sampled at a 1-s interval and miscellaneous other data. The measurements are referenced to the Mean Earth/Polar Axis Lunar Reference System (PDS Standards Reference, 2006). Topographic height measurements of SELENE and Chang'E-1 are referenced with respect to reference spheroids with radii of $1,737.4 \mathrm{~km}$ and $1,738 \mathrm{~km}$, respectively. The altimeter data time span provided by the SELENE mission is from December 30, 2007 to April 14, 2008, whereas the data provided by the Chang'E-1 mission is from November 27, 2007 to January 22, 2008. The respective laser altimeter data are used to compute single-satellite crossover statistics to evaluate their near-radial orbit and altimeter instrument time tag accuracy and the accuracy of the lunar topography models.

The SELENE lunar orbiter has a lower altitude $(100 \mathrm{~km})$ and is thus more sensitive to gravity perturbations, which may account for a less accurate orbit determination compared to that of the Chang'E-1 orbiter, which has a higher $(200 \mathrm{~km})$ mean orbital altitude. Conversely, Chang'E-1 is less sensitive to gravity perturbations, thus its data are less useful than SELENE for gravity eld model improvement. The tilting of the polar inclination and the low altitude cause the SELENE orbits to intermittently change from retrograde to prograde orbits. Given its higher altitude, the orbits of Chang'E-1 are presumably more stable. Unlike the SELENE orbiter, Chang'E-1 has no relay satellites for dedicated far side radiometric tracking, and thus it has a more degraded orbit accuracy than SELENE when orbiting over the lunar far side. In addition, the laser altimeter of Chang'E-1 laser is less accurate than that of SELENE.

Valid ranging measurements together with the precise or- 


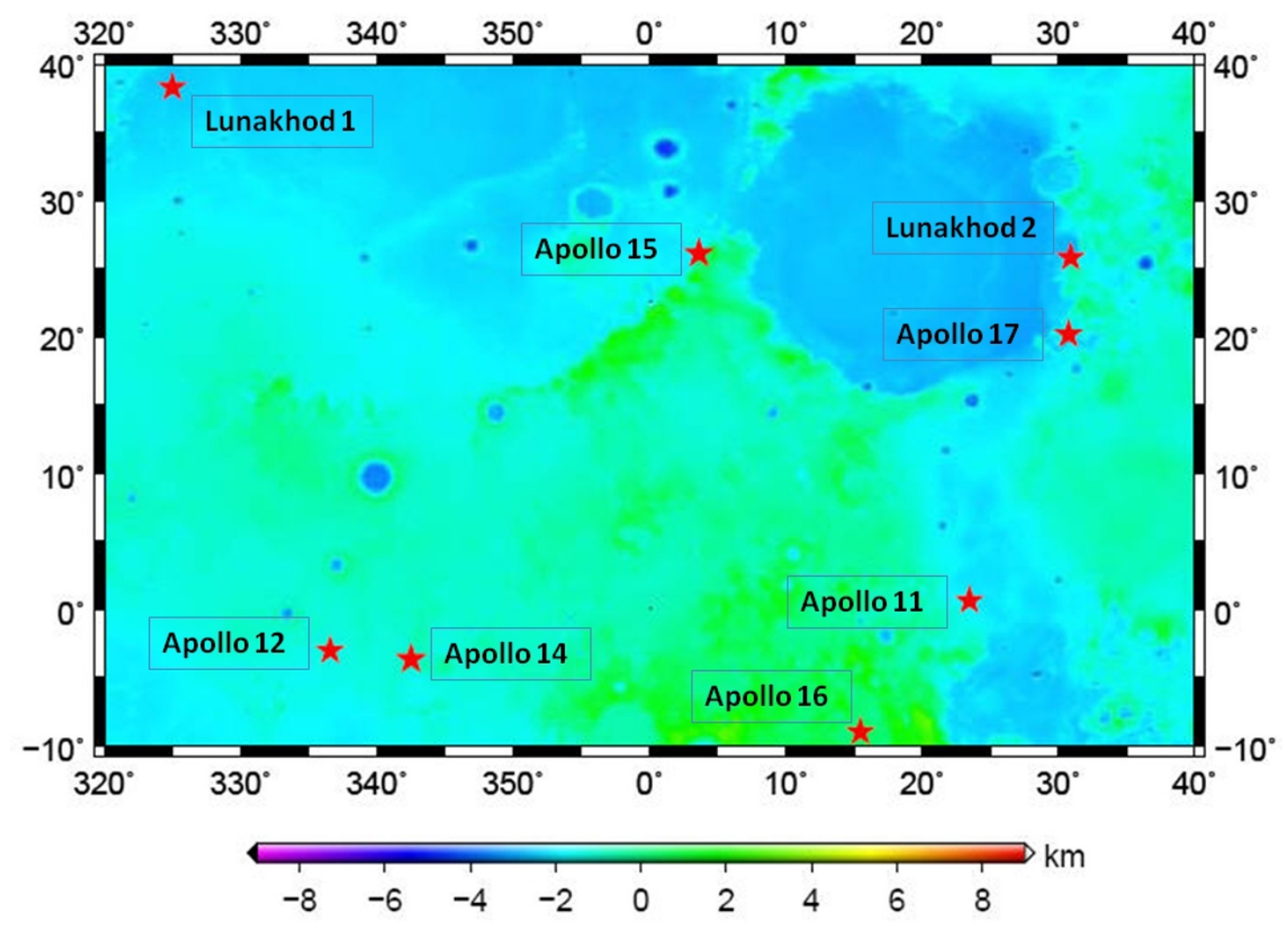

Fig. 1. Locations of the eight LLRR and ALSEP reference sites used in this study.

bits and attitude information yield topographic data (with 3.21 million and 6.77 million valid points for Chang'E-1 and SELENE, respectively, for the generation of the gridded topography models). These data, after subtracting their corresponding reference radius (i.e., 1,738 km for Chang'E-1 and 1,737.4 km for SELENE), were interpolated and assembled into a $0.0625^{\circ} \times 0.0625^{\circ}$ grid in a similar fashion (Archinal et al., 2007; Araki et al., 2009; Ping et al., 2009). The accuracies of these gridded datasets are evaluated by comparing them with the coordinates of the LLRR and ALSEP sites. Hereafter, the gridded topographies derived from the Chang'E-1 and SELENE missions are abbreviated as the Chang'E-1 (GT) and SELENE (GT), respectively.

\subsection{ULCN 2005 topography}

ULCN 2005 is a lunar control network derived from the previous Lunar Control Network developed by the RAND Corporation (Davies et al., 1994) and the Clementine Lunar Control Network (CLCN) (Edwards et al., 1996), in which most control point coordinates are established based on photogrammetric techniques with appropriate constraints on previous networks (Archinal et al., 2006). It includes the determination of the 3 -D positions of 272,931 points on the lunar surface. These points are interpolated and assembled into a $0.0625^{\circ} \times 0.0625^{\circ}$ grid with a reference radius of $1,737.4 \mathrm{~km}$. The topography model, which hereafter is abbreviated as ULCN2005 (GT), has been claimed to have radial uncertainties of several hundred meters. This will be discussed and verified later within the text.

\subsection{LLRR and ALSEP reference coordinates}

LLRRs are corner-cube retroreflector arrays that were installed on the Moon by the Lunakhod 2, Apollo 11, Apollo 14, and Apollo 15 missions, whereas ALSEPs, equipped with radio transmitters, were delivered by Apollo missions $12,14,15,16$, and 17 . The former sites have been observed by laser ranging for over three decades, while the ALSEP radio transmitters were observed between 1972 and 1974 through VLBI measurements (King et al., 1976). ALSEPs 14 and 15 are close enough to the retroreflectors to tie them together on the basis of photographs. Davies and Colvin (2000) combined the coordinate data; the LLRR coordinate uncertainties are $<3 \mathrm{~m}$, while the ALSEP errors are up to $10 \mathrm{~m}$ horizontally and $30 \mathrm{~m}$ vertically. With this level of accuracy, the reference coordinates serve as fundamental control points for the lunar coordinate system and can be used for lunar topography assessment (Dickey et al., 1994). Coordinates for LLRRs have recently been updated (Williams et al., 2008) and found to have uncertainties of $\leq 1 \mathrm{~m}$. Lunakhod 1 , a rover equipped with a retroflector that has been missing during the past four decades, was recently located on NASA Lunar Reconnaisance Orbiter images. Using those coordinates, its corner cube array was ranged by the Apache Point Observatory in April 2010. From an analysis of three nights of Apache Point data and aided by a stronger reflected signal than Lunakhod 2, Williams and Boggs (2010) found a radius of $1,734,929 \mathrm{~m}$ with a positioning uncertainty of $<1 \mathrm{~m}$. This site position is also included in the analysis, and its spatial location is displayed in Fig. 1. 


\section{Methods for Accuracy Assessment}

Two methods were employed to assess the accuracy of the topography models. The rst involves the usage of the coordinates of the LLRR and ALSEP sites as the external reference control points for checking against the derived topography models. This is referred to as the external accuracy assessment. The second method uses only the laser altimeter data for conducting crossover analysis. This is referred to as the internal accuracy assessment.

\subsection{External accuracy assessment}

Given the reference coordinates of the LLRR and ALSEP sites, the derived topographies, which are the heights referenced to their respective radii re-sampled on a $0.0625^{\circ} \times$ $0.0625^{\circ}$ grid size, can then be externally assessed through bilinear interpolation.

Bilinear interpolation, which is widely used in surface and terrain modeling, is a geometrical method that relates 2-D planar coordinates, $(x, y)$, which correspond to the longitude and latitude, $(\lambda, \phi)$, with a planar approximationin this case to the height of a particular place of interest, $h$. The general mathematical form can be described mathematically as

$$
h=a_{0}+a_{1} x+a_{2} y+a_{3} x y
$$

where $a_{0}, a_{1}, a_{2}, a_{3}$ are sets of coef cients to be determined (Li et al., 2005). At least four neighboring points with heights are required to obtain a unique solution. In our case, gridded heights of the topography models were used as observations for the determination of the sets of coef cients. This is deployed in approximating the height at a desired location.

Since each gridded height of the topography models provides no valid means to indicate the accuracy, each height can only be assumed to be of the same accuracy. In many cases, the coordinates of the LLRR and ALSEP reference sites are not located near the center of the interpolated grid points, but near the boundary of those points. Simple bilinear interpolation based only on four gridded points will depend heavily on the two nearest points of the boundary to obtain a height in the desired location. For this reason, a search within a certain distance between the coordinates of a LLRR or ALSEP site and neighboring gridded points was conducted instead of the former approach. A search distance of $0.09^{\circ}$, which corresponds to approximately $3 \mathrm{~km}$ on the moon, was used to nd the nearest neighboring gridded points (i.e. $>4$ points) as the observations for the determination of a set of coef cients of the bilinear interpolation with redundancy. Also, because different reference radii were applied to different topography models while the altitude information of the LLRR and ALSEP sites were in the form of radii with respect to the center of mass of the moon, each interpolated height is added to their corresponding reference radius. Hence, the interpolated radii were subsequently compared to those radii of the reference LLRR and ALSEP sites.

\subsection{Internal accuracy assessment}

SELENE and Chang'E-1 laser altimeter data, which enables full coverage of measurements on the far side and the polar areas of the Moon, in contrast to previous lunar missions, provide the fundamental data source for deriving the lunar topography. The data from the new missions has improved the far-side topography, and polar altimetry is now possible. Despite its advantage in the spatial distribution of the data, a consideration of possible error sources when these data are used for orbit determination and geophysical mapping reveals several disadvantages.

For the purpose of precise orbit determination and the recovery of geophysical parameters of interest, crossover analysis was developed to obtain relevant measurements separated in time at the same geographic location where ascending and descending ground tracks of the satellite orbits intersect (Shum et al., 1990). Crossover analysis is routinely used for the evaluation of satellite orbit accuracy and the accuracy of Earth satellite altimetry-observed sea surface topography height measurements. Over the Moon, terrain roughness and pointing accuracy of the spacecraft as well as any constant or periodic pointing biases can significantly affect the accuracy of the geolocation of the laser altimeter measurements.

By taking the difference between the measured height, $h$, of the same location at time tag $t_{i}$ and time tag $t_{j}$, the crossover measurements are computed and represented by

$$
\begin{aligned}
\Delta h\left(t_{i}, t_{j}\right)= & h\left(t_{i}\right)-h\left(t_{j}\right) \\
= & b+\dot{h} \tau+\sum_{k=1}^{\ell}\left[S_{k} \sin \omega_{k}\left(t-t_{0}\right)\right. \\
& \left.+C_{k} \cos \omega_{k}\left(t-t_{0}\right)\right]-\Delta f R_{\mathrm{e}} \sin ^{2} \phi
\end{aligned}
$$

where $b$ is a constant bias, $\dot{h}$ is the time rate of change of height, $\tau$ is the time tag bias, $\omega_{k}$ is a speci ed frequency, $S_{k}$ and $C_{k}$ are amplitudes of trigonometric functions, $t$ and $t_{0}$ are the time epoch of observations and initial reference epoch, $R_{\mathrm{e}}$ is the mean radius of the best tting ellipsoid, $\phi$ is the spherical latitude, and $\Delta f$ is the reciprocal of the attening of the ellipsoid. $b, \tau, S_{k}, C_{k}$, and $\Delta f$ are the parameters to be estimated. Since the orbit errors are dominated by errors at a frequency of once per orbital revolution, $\ell$ is set to 1. Note that each $h\left(t_{i}\right)$ and $h\left(t_{j}\right)$ in Eq. (2) contains altitude error, orbit errors including gravity-induced orbit error, time tag bias of the altimeter instrument, and other unmodeled errors. The computed crossover measurement, $\Delta h\left(t_{i}, t_{j}\right)$, will hence eliminate any non-temporal and very long wavelength components and leave temporal change components at the crossover location when it is measured. Nevertheless, the Moon is, in essence, less complicated when compared to the Earth because of the absence of the time-varying oceanic surface. This, to a large extent, eliminates most temporal change components, except the variable component of the orbital error (both horizontal and radial) due to various sources, including the errors in the a priori lunar gravity eld model and others, such as the time tag error.

In reality, it is rare that altimeter measurements are available precisely at the crossover times. The single-satellite crossover locations are found through the prediction of nominal crossovers followed by the interpolation of discrete sub-satellite points projected from satellite orbits (Shum, 1982; Schutz et al., 1982; Shum et al., 1990). Hence, the computed crossover height differences at different locations permit an internal accuracy assessment. It should be noted that due to the rough terrain, the acute angles at 
Table 1. The radial differences in meters, $\Delta r$, between the gridded topography (GT) models, Chang'E-1 (GT), SELENE (GT), ULCN2005 (GT), and the LLRR and ALSEP reference sites, and their normalized differences, $T$ (dimensionless statistical test values), computed by dividing $\Delta r$ with to their claimed radial uncertainties of $31 \mathrm{~m}, 50 \mathrm{~m}$, and $300 \mathrm{~m}$, respectively. A bias correction of $+150 \mathrm{~m}$ was added to Chang'E-1 (GT). The Lunakhod 1 site annotated with an asterisk $\left(^{*}\right)$ is a newly recovered reference site based on lunar laser ranging (Williams and Boggs, 2010). The values in the brackets are RMS radial difference when this newly recovered site is added in the analysis.

\begin{tabular}{lcrrrrr}
\hline Reference sites & \multicolumn{2}{c}{ Chang'E-1 $(\mathrm{GT})$} & \multicolumn{2}{c}{ SELENE (GT) } & \multicolumn{2}{c}{ ULCN2005 (GT) } \\
\cline { 2 - 6 } & $\Delta r(\mathrm{~m})$ & \multicolumn{1}{c}{$T$} & $\Delta r(\mathrm{~m})$ & \multicolumn{1}{c}{$T$} & $\Delta r(\mathrm{~m})$ & $T$ \\
\hline Lunakhod 1 (LLRR) & 3 & 0.11 & 9 & 0.19 & 6 & 0.02 \\
Lunakhod 2 (LLRR) & 452 & 14.60 & 98 & 1.96 & 750 & 2.50 \\
Apollo 11 (LLRR) & 17 & 0.56 & 14 & 0.29 & 217 & 0.72 \\
Apollo 12 (ALSEP) & -45 & -1.45 & -35 & -0.71 & 74 & 0.25 \\
Apollo 14 (LLRR) & 24 & 0.77 & 43 & 0.86 & -145 & -0.49 \\
Apollo 14 (ALSEP) & 16 & 0.51 & 36 & 0.72 & -153 & -0.51 \\
Apollo 15 (LLRR) & 3 & 0.10 & -83 & -1.66 & 886 & 2.95 \\
Apollo 15 (ALSEP) & 2 & 0.05 & -83 & -1.66 & 879 & 2.93 \\
Apollo 16 (ALSEP) & -40 & -1.30 & -22 & -0.45 & 347 & 1.16 \\
Apollo 17 (ALSEP) & 120 & 3.87 & -40 & -0.80 & 511 \\
\hline RMS radial differences (m) & \multicolumn{2}{c}{$157(150)$} & $58(55)$ & & $537(510)$ \\
\hline
\end{tabular}

crossover points (due to dif culties in computing crossovers because of the polar orbital inclination), pointing error (i.e., the laser range measurements are not along the nadir position), and incomplete global coverage will increase the crossover residual root mean square (RMS), which may not be totally dominated by orbit and instrument errors. This may be largely because of the horizontal error-for example because the crossovers were computed using altimeter range measurements, which are not exactly in the nadir directions.

The crossover residuals are presented before and after empirical adjustment of the radial orbit error, assuming a model that the orbit errors are dominated by errors at a frequency of once per orbital revolution (Shum et al., 1990). The adjustment has been proven effective to remove orbit errors for Earth radar altimetry satellites.

\section{Results and Discussion}

For external accuracy assessment, the radial differences of the gridded topographies, Chang'E-1 (GT), SELENE (GT), and ULCN2005 (GT) from the LLRR and ALSEP reference sites are computed in association with their corresponding statistics (Table 1). A bias correction of $+150 \mathrm{~m}$ has been added to the interpolated radii of Chang'E-1 before conducting the external accuracy assessment. This bias is also con rmed when the mean radius of Chang'E-1 obtained from spherical harmonic decomposition is intercompared with the SELENE mean radius.

Normalized differences, $T$, are the radial differences divided by their claimed radial uncertainties (Table 1). Normalized differences $>2.576$ represent a radial difference beyond the statistical value expected at the $99 \%$ con dence level, in which the expectation of the radial differences should be equal to zero if the topographies contain no errors with respect to the LLRR and ALSEP reference sites. The radial differences among the gridded topographies at Apollo 11, 12, 14, and newly recovered Lunakhod 1 are shown to be in agreement with each other in terms of their normalized differences. There are relatively at surfaces over the regions at those sites. Apollos 11 and 12 are on
Mare surfaces; Apollo 14 is on the rougher Fra Mauro formation; Lunakhod 1 is on Mare Imbrium. The other sites have more complex topography. At the Lunakhod 2 site, the radial differences among the gridded topographies are larger, possibly because the site (Fig. 1) is near the southern rim of the crater Le Monnier. The Apollo 15 site is near the rim of the Imbrium basin, Apollo 16 was on an uplands plain, and the Apollo 17 site is in the Taurus-Littrow valley. The resulting radial differences (not shown here) are nearly the same when compared with the more recent LLRR site coordinates tabulated by Williams et al. (2008) because the updated values deviate by only $1 \mathrm{~m}$ from the old ones in the radial component. Repeatability analysis, which computes the deviation of the surface using gridded data near the sites, was also conducted to validate the computed radial differences.

For the internal accuracy assessment, the crossover differences were computed from Chang'E-1 and SELENE laser altimeter data. Locations of crossovers for both Chang'E-1 and SELENE concentrate near the pole because of near-polar inclination. Their respective histograms for the rst-month data are displayed in Figs. 2 and 3. Both histograms are shown to be normally distributed, where the dispersion of the crossover difference for Chang'E-1 is larger than that of SELENE, despite different numbers of crossovers. This is also validated from the spatial distribution of the magnitude of crossover differences in lunar polar regions (Fig. 4). The number of crossovers in Chang'E-1 is considerably less than that of SELENE, because its measurements are irregularly sampled.

The crossover residuals are computed using all of the available measurements covering the laser altimeter data span and the crossover residual statistics are shown in Table 2. It shows that the longer the time span of data, the larger the RMS crossover differences. This result may be due to the horizontal orbit error and the mis-pointing of the altimeter instrument (Goossens et al., 2008) and potentially due to orbital revolution error in the long period (longer than crossover times). The RMS crossover differences of Chang'E-1 are threefold larger than those of SELENE, re- 


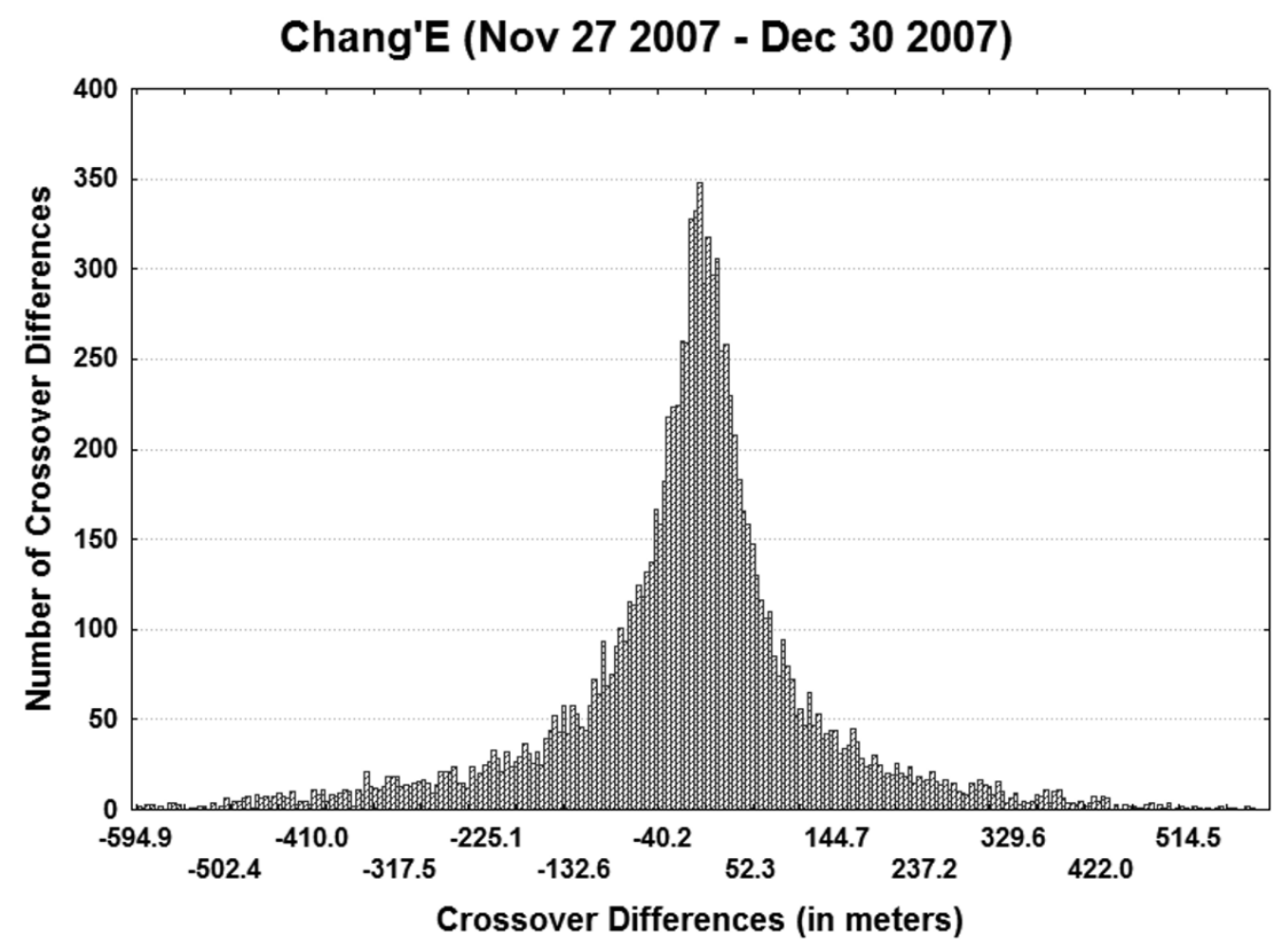

Fig. 2. Histogram of the crossover differences for Chang'E-1 between 27 November and 30 December 2007.

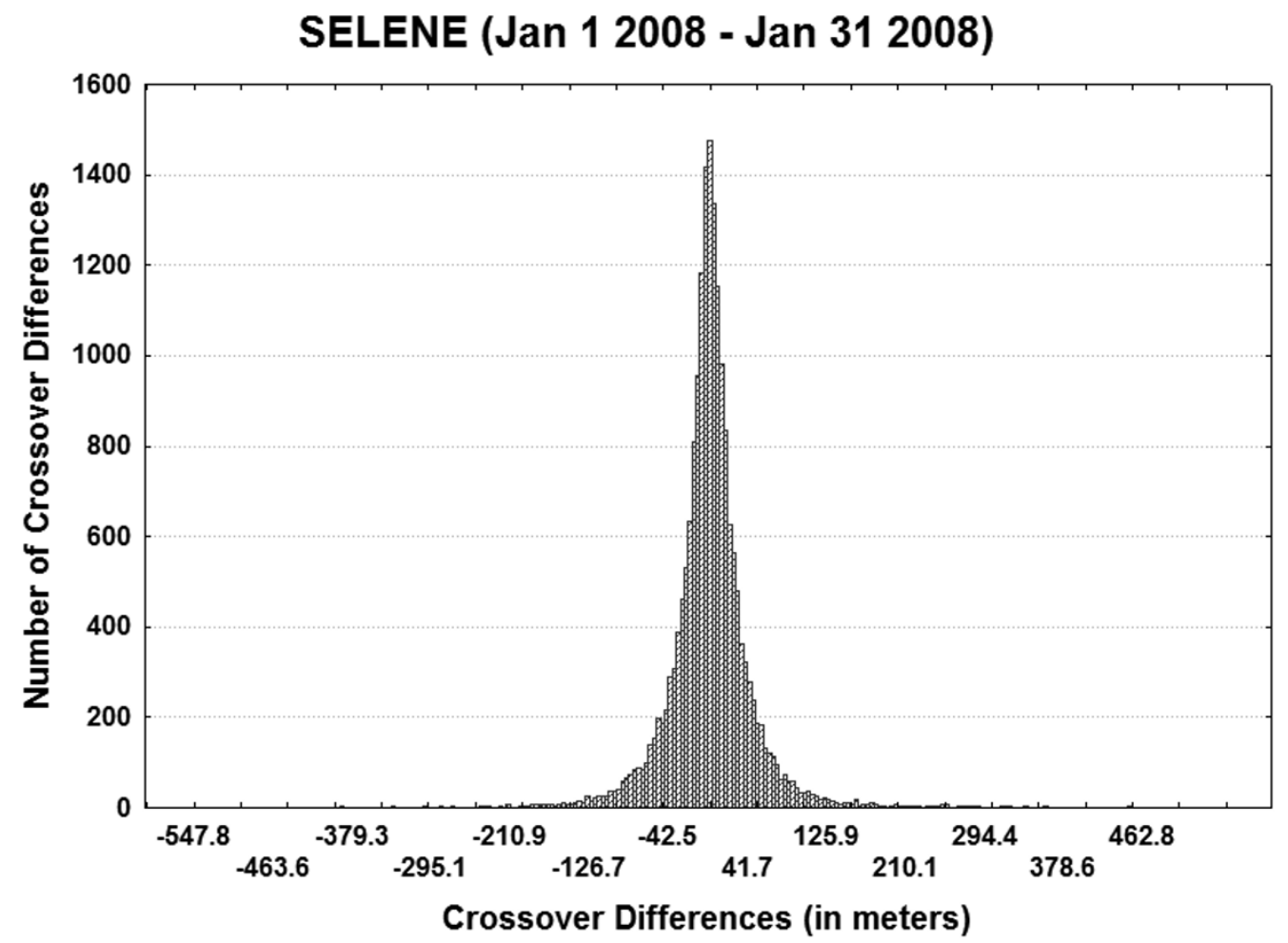

Fig. 3. Histogram of the crossover differences for SELENE between 1 January and 31 January 2008. 
Chang'E (North>85)

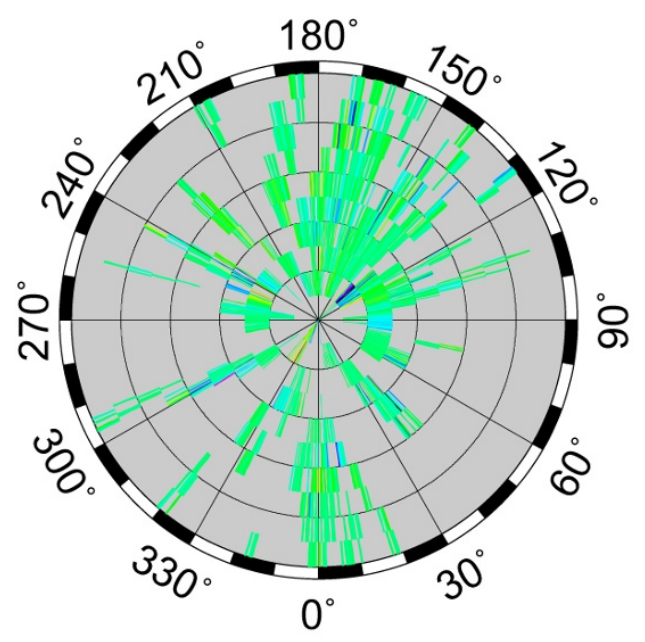

SELENE (North>85)

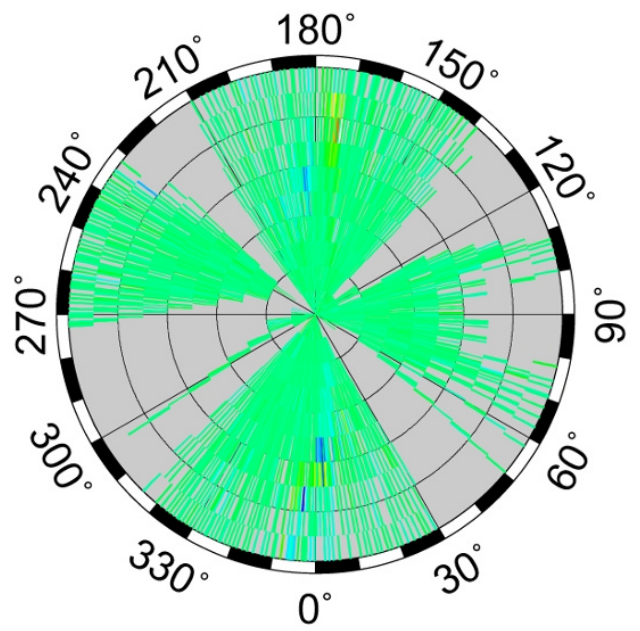

Chang'E (South<-85)

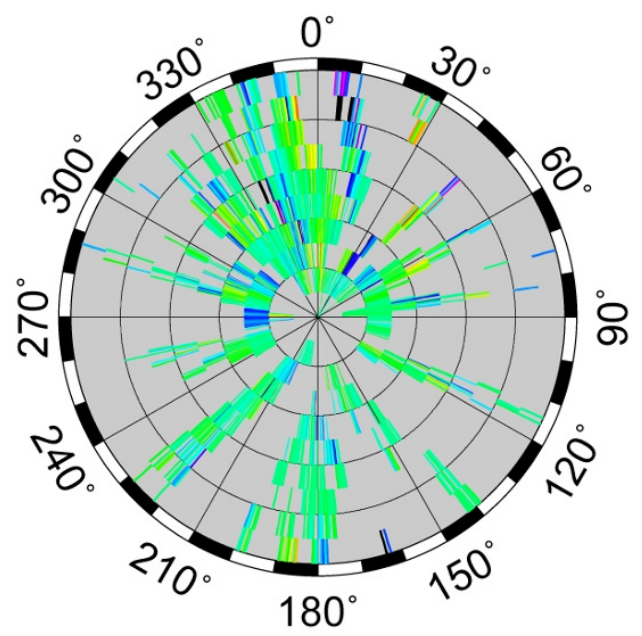

SELENE (South<-85)

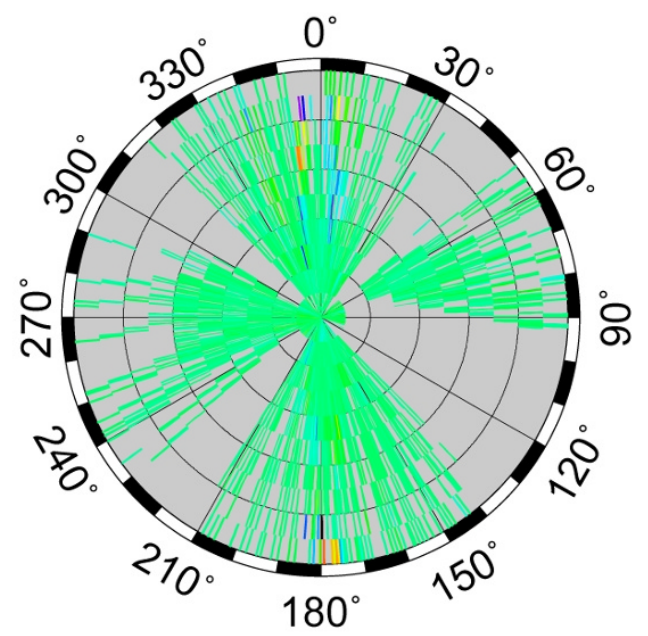

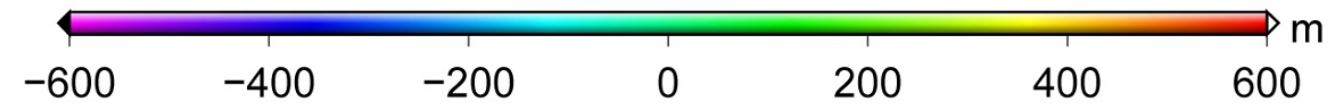

Fig. 4. Crossover differences of Chang'E-1 and SELENE in the polar regions.

Table 2. The crossover statistics for Chang'E-1 and SELENE laser altimeter data. Note that RMS (before) and RMS (after) represents RMS crossover difference before and after crossover adjustment.

\begin{tabular}{lccc}
\hline Data time span & No. of crossovers & RMS (before) (m) & RMS (after) (m) \\
\hline Chang'E-1 (27 Nov-30 Dec 2007) & 10,882 & 152.8 & 84.7 \\
Chang'E-1 (27 Nov-22 Jan 2008) & 22,941 & 335.5 & 206.3 \\
SELENE (1 Jan-31 Jan 2008) & 19,194 & 54.3 & 37.0 \\
SELENE (1 Jan-14 April 2008) & 230,635 & 124.6 & 68.5 \\
\hline
\end{tabular}

gardless of either before or after the adjustment (empirical orbit adjustment assuming that the orbit error is dominated by a once-per-orbital revolution error), as also revealed in the histograms (Figs. 2 and 3). The larger Chang'E-1 crossover residual RMS (than SELENE) may also be due to the fact that the Chang'E-1 laser altimeter $( \pm 5 \mathrm{~m})$ has a worse precision than that of SELENE $( \pm 4 \mathrm{~m})$. However, the horizontal orbit error and the mis-pointing of the altime- ter instruments are the main causes of crossover position error, which can be severe at some crossover locations, as evidenced in Fig. 4.

The RMS crossover differences after adjustment are merely a bit larger than the RMS radial differences for the external accuracy, as shown in Table 1 . This implies that the results from both the external and internal accuracy assessment are consistent with each other, allowing us to set upper 
bound errors of $200 \mathrm{~m}$ and $70 \mathrm{~m}$ for the Chang'E-1 (GT) and SELENE (GT), respectively. Given the RMS radial differences of ULCN2005 (GT) in Table 1, Chang'E-1 (GT) and SELENE (GT) represent an improvement by factors of 2.5 and 8, respectively, when compared to ULCN2005 (GT).

Noda et al. (2009) presented a new external accuracy assessment methodology in which the in-situ laser altimeter data near the LLRR reference sites are directly used for providing an accuracy assessment. They found that SELENE topographic accuracy, directly inferred from the laser altimeter data, was at a 40-m level. This result further conrms our accuracy estimates when those sites with signi cant radial differences are ignored in our external accuracy assessment.

\section{Conclusion}

This paper reports both the external and internal accuracy assessment for the topography models derived from recent space exploration missions-Chang'E-1 and SELENE. Utilization of the coordinates of the LLRR and the radiotracked ALSEP sites located around the equatorial region (external) along with the formation of crossovers from the laser altimeter data records that are mainly located in polar region (internal) ensure a global lunar spatial coverage for comprehensive accuracy assessment.

The external accuracy assessment shows that the accuracy of the topography derived from SELENE is approximately $58 \mathrm{~m}$, whereas the Chang'E-1 and the ULCN2005 topography are comparatively larger (i.e. approx. $157 \mathrm{~m}$ and approx. $537 \mathrm{~m}$, respectively). These magnitudes of RMS radial and crossover differences, based on the two respective assessments, are comparable to each other. This result substantiates the accuracy of the two derived topography models. The estimates on the accuracy of Chang'E-1 and SELENE topographies based on both accuracy assessments, which permit the establishment of the conservative accuracy estimates of $200 \mathrm{~m}$ and of $70 \mathrm{~m}$, represent signi cant improvements by factors of 2.5 and 8 , respectively, when compared to the ULCN2005 topography.

The lunar topography models derived here have an improved accuracy. Continuous efforts on reducing errors in the laser altimeter data records in the near future will further improve the accuracy and resolution of the lunar topography, and hence, our understanding of the interior of the Moon and its origin.

Acknowledgments. We acknowledge JAXA via the SELENE project, and the Chang'E-1 project for providing the lunar data products for this study. The SELENE foreign co-investigators (C. K. Shum, J. Ping, and J. Oberst) are very grateful for JAXA's support. The Ohio State University authors appreciate funding from The Ohio State University, which has made this study possible. A portion of the research described in this paper was carried out at the Jet Propulsion Laboratory of the California Institute of Technology, under a contract with the National Aeronautics and Space Administration. We thank H. Baki Iz of Hong Kong Polytechnic University for useful comments that helped improve the manuscript.

\section{References}

Araki, H., S. Tazawa, H. Noda, T. Tsubokawa, N. Kawano, and S. Sasaki, Observation of the lunar topography by the laser altimeter LALT on board Japanese lunar explorer SELENE, Adv. Space Res., 42, 317-322, 2008.

Araki, H., S. Tazawa, H. Noda, Y. Ishihara, S. Goossens, S. Sasaki, N. Kawano, I. Kamiya, H. Otake, J. Oberst, and C. Shum, Lunar global shape and polar topography derived from Kaguya-LALT laser altimetry, Science, 323(5916), 897-900, 2009.

Archinal, B. A., M. R. Rosiek, R. L. Kirk, and B. L. Redding, The uni ed lunar control network 2005, U.S. Geological Survey Open-File Report 2006-1367 Version 1.0, 2006.

Archinal, B. A., M. R. Rosiek, R. L. Kirk, T. L. Hare, and B. L. Redding, Report on the nal completion of the uni ed lunar control network 2005 and lunar topographic model, Lunar Planet. Sci., 37, \#1904, 2007.

Davies, M. E. and T. R. Colvin, Lunar coordinates in the regions of the Apollo landers, J. Geophys. Res., 105(E8), 20277-20280, 2000.

Davies, M. E., T. R. Colvin, D. L. Meyer, and S. Nelson, The uni ed lunar control network; 1994 version, J. Geophys. Res., 99(E11), 2321123214, 1994.

Dickey, J. O., P. L. Bender, J. E. Faller, X. X. Newhall, R. L. Ricklefs, J. G. Ries, P. J. Shelus, C. Veillet, A. L. Whipple, J. R. Wiant, J. G. Williams, and C. F. Yoder, Lunar laser ranging: A continuing legacy of the Apollo program, Science, 265(5171), 482-489, 1994.

Edwards, K. E., T. R. Colvin, T. L. Becker, D. Cook, M. E. Davies, T. C. Duxbury, E. M. Eliason, E. M. Lee, A. S. McEwen, H. Morgan, M. S. Robinson, and T. Sorensen, Global digital mapping of the Moon, Lunar Planet. Sci., XXVII, 335-336, 1996.

Goossens, S., K. Matsumoto, Y. Ishihara, Q. Liu, F. Kikuchi, H. Noda, T. Iwata, N. Namiki, and H. Hanada, Analysis of tracking data and results from Kaguya (SELENE) satellites for lunar gravity eld estimation, AGU Fall Meeting 2008, abstract P31B-1400, 2008.

Kikuchi, F., Q. Liu, H. Hanada, N. Kawano, K. Matsumoto, T. Iwata, S. Goossens, K. Asari, Y. Ishihara, S. Tsuruta, T. Ishikawa, H. Noda, N. Namiki, N. Petrova, Y. Harada, J. Ping, and S. Sasaki, Pico-second accuracy VLBI of the two sub-satellites of SELENE (KAGUYA) using multi-frequency and same beam methods, Radio Sci., 44, RS2008, doi:10.1029/2008RS003997, 2009.

King, R. W., C. C. Counselman, and I. I. Shapiro, Lunar dynamics and selenodesy: results from analysis of VLBI and laser data, J. Geophys. Res., 81(35), 6251-6256, 1976.

Li, Z., Q. Zhu, and C. Gold, Digital Terrain Modeling: Principles and Methodology, 323 pp., CRC Press, Boca Raton, FL, 2005.

Namiki, N., H. Hanada, T. Tsubokawa, N. Kawano, M. Ooe, K. Heki, T. Iwata, M. Ogawa, and T. Takano, Selenodetic experiments of SELENE: Relay subsatellite, differential VLBI, and laser altimeter, $A d v$. Space Res., 23(11), 1817-1820, 1999.

Namiki, N., T. Iwata, K. Matsumoto, H. Hanada, H. Noda, S. Goossens, M. Ogawa, N. Kawano, K. Asari, S. Tsuruta, Y. Ishihara, Q. Liu, F. Kikuchi, T. Ishikawa, S. Sasaki, C. Aoshima, K. Kurosawa, S. Sugita, and T. Takano, Farside gravity eld of the Moon from four-way Doppler measurements of SELENE (Kaguya), Science, 323(5916), 900-905, 2009

Noda, H., H. Araki, S. Goossens, Y. Ishihara, K. Matsumoto, S. Tazawa, N. Kawano, and S. Sasaki, Illumination conditions at the lunar polar regions by KAGUYA (SELENE) laser altimeter, Geophys. Res. Lett. 35, L24203, doi:10.1029/2008GL035692, 2008.

Noda, H., H. Araki, S. Tazawa, S. Goossens, Y. Ishihara, K. Matsumoto, N. Kawano, S. Sasaki, I. Kamiya, J. Oberst, and C. K. Shum, KAGUYA (SELENE) laser altimeter (LALT): one year in orbit, European Geosciences Union General Assembly 2009, Vienna, Austria, April 19-24, 2009.

PDS Standards Reference, Version 3.7, http://pds.jpl.nasa.gov/ documents/sr, 2006.

Ping, J. S., Q. Huang, J. G. Yan, J. F. Cao, G. S. Tang, and R. Shu, Lunar topographic model CLTM-s01 from Chang'E-1 laser altimeter, Sci. China G, 52(7), 1104-1115, 2009.

Schutz, B. E., B. D. Tapley, and C. Shum, Evaluation of the SEASAT altimeter time tag bias, J. Geophys. Res., 87(C5), 3239-3245, 1982.

Shum, C. K., Altimeter methods for satellite geodesy, PhD dissertation, The University of Texas at Austin, 1982.

Shum, C. K., B. H. Zhang, B. E. Schutz, and B. D. Tapley, Altimeter crossover methods for precision orbit determination and the mapping of geophysical parameters, J. Astronaut. Sci., 38, 355-368, 1990.

Sjogren, W. L. and W. R. Wollenhaupt, Lunar shape via the Apollo laser altimeter, Science, 179(4070), 275-278, 1973.

Smith, D. E., M. T. Zuber, G. A. Neumann, and F. G. Lemoine, Topography of the Moon from the Clementine lidar, J. Geophys. Res., 102(E1), 
1591-1611, 1997.

Wieczorek, M. A., The gravity and topography of the terrestrial planets, Treatise Geophys., 10, 165-206, 2007.

Wieczorek, M. A., B. L. Jolliff, A. Khan, M. E. Pritchard, B. P. Weiss, J. G. Williams, L. L. Hood, K. Righter, C. R. Neal, C. K. Shearer, I. S. McCallum, S. Tompkins, B. R. Hawke, C. Peterson, J. J. Gillis, and B. Bussey, Chapter 3. The constitution and structure of the lunar interior, in New Views of the Moon, edited by B. L. Jolliff, M. A. Wieczorek, C. K. Shearer, and C. R. Neal, Rev. Mineral. Geochem., 60, 221-364, Chantilly, VA, 2006.

Williams, J. G. and D. H. Boggs, Private Communication, Jet Propulsion Laboratory, California Institute of Technology, 2010.
Williams, J. G., D. H. Boggs, and W. M. Folkner, DE421 lunar orbit, physical librations, and surface coordinates, JPL Memorandum IOM 335-JW, DB, WF-20080314-001, March 14, ftp://ssd. jpl.nasa.gov/pub/eph/planets/ioms/de421_Moon_coord_iom.pdf, 2008.

Zuber, M. T., D. E. Smith, F. G. Lemoine, and G. A. Neumann, The shape and internal structure of the Moon from the Clementine mission, Science, 266(5192), 1839-1843, 1994.

H. S. Fok (e-mail: fok.8@buckeyemail.osu.edu), C. K. Shum, Y. Yi, H. Araki, J. Ping, J. G. Williams, G. Fotopoulos, H. Noda, S. Goossens, Q. Huang, Y. Ishihara, K. Matsumoto, J. Oberst, and S. Sasaki 